02

\title{
О расщеплении полосы проводимости ртути в жидком состоянии
}

\author{
(C) А.И. Киселев \\ Институт химии твердого тела Уральского отделения РАН, \\ 620990 Екатеринбург, Россия \\ e-mail: kis1771@yandex.ru
}

Поступила в редакцию 22.03.2018 г.

Представлены результаты расчета электронных характеристик вкладов в оптическую проводимость ртути при комнатной температуре, установленные из процедуры сравнения модельного спектра оптической проводимости с данными эксперимента. Показано, что экспериментальный спектр оптической проводимости жидкой ртути может быть описан только с привлечением представления о возможности расщепления полосы проводимости.

DOI: $10.21883 /$ OS.2018.08.46359.84-18

\section{Введение}

Представление о расщеплении полосы проводимости ртути в жидком состоянии было введено Моттом в работе [1]. Причину существенной расходимости экспериментальных данных по электропроводности с модельными оценками подхода Друде он объяснял наличием существенного понижения плотности состояний в области поверхности Ферми в энергетическом спектре электронов ртути.

Экспериментальные данные различных методов подтверждают гипотезу Мотта о наличии псевдощели в энергетическом спектре ртути. В работах [2,3] методом ультрафиолетовой фотоэмиссионной спектроскопии исследована плотность состояний ртути вблизи уровня Ферми. При этом экспериментальный спектр электронов сравнивался со спектром модели почти свободных электронов (ПСЭ). Показано, что плотность состояний на уровне Ферми заметно меньше, чем это следует из модели почти свободных электронов.

В работах [4-6] по данным углового распределения гамма-квантов при аннигиляции позитронов, облучающих металл, изучали ртуть и разбавленные амальгамы индия и натрия. Из результатов этих исследований также сделан вывод о понижении плотности состояний на уровне Ферми. Отмечалось, что добавление $4 \%$ натрия еще более снизило эту плотность.

Для ртути также характерно наличие фазового перехода металл-изолятор. Такой переход в ней наблюдали экспериментально [7,8]. При низких температурах ее электропроводность имеет явный металлический характер. При высоких температурах проводимость падает на несколько порядков и приближается по величине к проводимости полупроводников. Позднее Хафнером [9] с помощью первопринципного пакета VASP была рассчитана плотность электронных состояний для широкого диапазона плотностей ртути, и было найдено, что при плотности ртути $8.8 \mathrm{~g} / \mathrm{cm}^{-3}$, в области которой совершается переход металл-изолятор, в плотности состояний образуется щель.
Исследование оптических свойств также позволяет судить о степени отклонения характеристик проводимости ртути от модели почти свободных электронов. Анализ экспериментально установленной зависимости оптический проводимости от частоты света свидетельствует о несоответствии с моделью ПСЭ [10,11].

Здесь при анализе данных оптического эксперимента для ртути используется методика работы [12], в которой при изучении спектра оптической проводимости расплава алюминия было высказано предположение, что расщепление полосы проводимости алюминия, обеспечивающее свойства сверхпроводимости при низких температурах, сохраняется вплоть до температуры плавления. Отмечалось, что для алюминия в жидком состоянии количество электронов в верхней (наиболее широкой) части полосы проводимости минимально, или их нет вообще. При этом вклад в оптическую проводимость от проводимости, описываемой моделью Друде, близок к нулю. Стационарную проводимость алюминия, наблюдаемую в опыте, обеспечивают электроны, находящиеся на уровнях вблизи полосы проводимости. Этот факт можно трактовать в духе предположения Мотта о возможности существовании внутри полосы проводимости запрещенной области, т.е. говорить о расщеплении полосы проводимости в расплаве алюминия.

\section{Результаты анализа данных оптического эксперимента}

На основе процедуры сравнения экспериментальных спектров оптической проводимости с модельными спектрами [13] была сформирована экспертная система, на базе которой производилось определение характеристик электронных состояний, составляющих эти спектры. В частности, это позволяет привлекать к анализу полученные ранее экспериментальные данные. В настоящей работе экспертная система применена к представленным на рис. 1 экспериментальным данным [11] для спектра оптической проводимости ртути при комнатной температуре. В работе [11] совмещены результаты работы [14], 
Электронные характеристики модельного спектра оптической проводимости в жидкой ртути при $m^{*}=0.94$

\begin{tabular}{|c|c|c|c|c|c|c|c|c|c|}
\hline \multirow{2}{*}{$j$} & $\Lambda . \mathrm{VV}$ & $\omega_{p j}$ & $\omega_{0 j}$ & \multirow{2}{*}{$z_{j}$} & \multirow{2}{*}{$j$} & $\wedge \quad \mathrm{V}$ & $\omega_{p j}$ & $\omega_{0 j}$ & \multirow{2}{*}{$z_{j}$} \\
\hline & $\Delta_{j}, \mathrm{eV}$ & \multicolumn{2}{|c|}{$\times 10^{-15}, \mathrm{~s}^{-1}$} & & & $\Delta_{j}, \mathrm{ev}$ & \multicolumn{2}{|c|}{$\times 10^{-15}, \mathrm{~s}^{-1}$} & \\
\hline 0 & 0 & 0.0000 & 0.0100 & 0.0010 & 9 & 1.6627 & 2.6670 & 0.0632 & 0.0550 \\
\hline 1 & 0.4245 & 8.8961 & 0.1813 & 0.6121 & 10 & 1.7612 & 2.2193 & 0.0272 & 0.0381 \\
\hline 2 & 0.6181 & 4.8419 & 0.1667 & 0.1813 & 11 & 1.8635 & 2.5561 & 0.0283 & 0.0505 \\
\hline 3 & 0.7585 & 4.9115 & 0.1643 & 0.1866 & 12 & 1.9554 & 1.9724 & 0.0308 & 0.0301 \\
\hline 4 & 0.9014 & 4.9123 & 0.1606 & 0.1866 & 13 & 2.1115 & 2.7201 & 0.0655 & 0.0572 \\
\hline 5 & 1.0456 & 4.6951 & 0.1584 & 0.1705 & 14 & 2.3039 & 2.5517 & 0.0668 & 0.0504 \\
\hline 6 & 1.2015 & 4.1455 & 0.1563 & 0.1329 & 15 & 2.5456 & 1.9637 & 0.0862 & 0.0298 \\
\hline 7 & 1.3725 & 3.9155 & 0.1498 & 0.1186 & 16 & 2.8677 & 2.6398 & 0.0782 & 0.0539 \\
\hline 8 & 1.5338 & 3.7578 & 0.1285 & 0.1092 & & & & & \\
\hline
\end{tabular}

где измерены оптические характеристики в диапазоне энергии фотонов от 0.5 до $2 \mathrm{eV}$, и результаты работы [15] с рабочим диапазоном от 1.8 до $3 \mathrm{eV}$. Эти результаты бы-

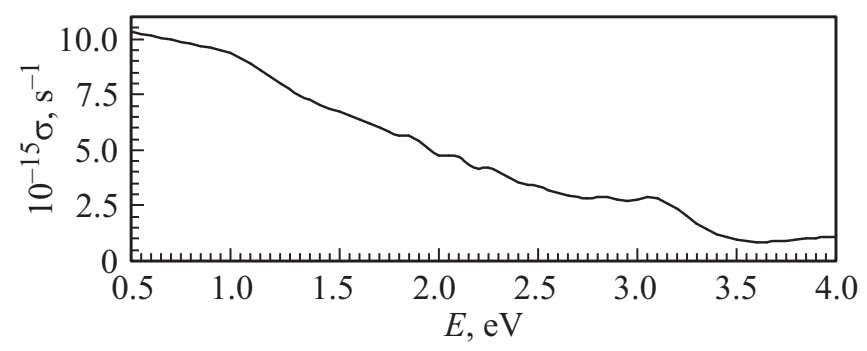

Рис. 1. Спектр оптической проводимости $\sigma$ ртути [10] и [14].

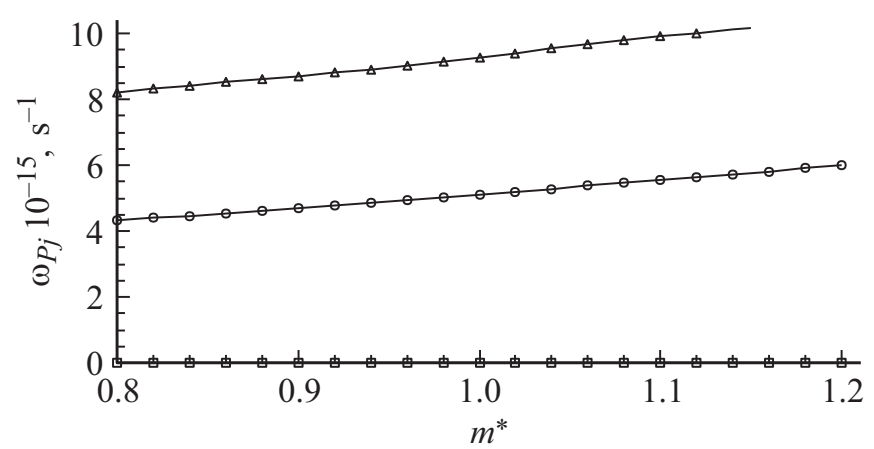

Рис. 2. Частоты плазменных колебаний ртути: $\square-\omega_{p 0}, \Delta-$ $\omega_{p 1}, \circ-\omega_{p 2}$.

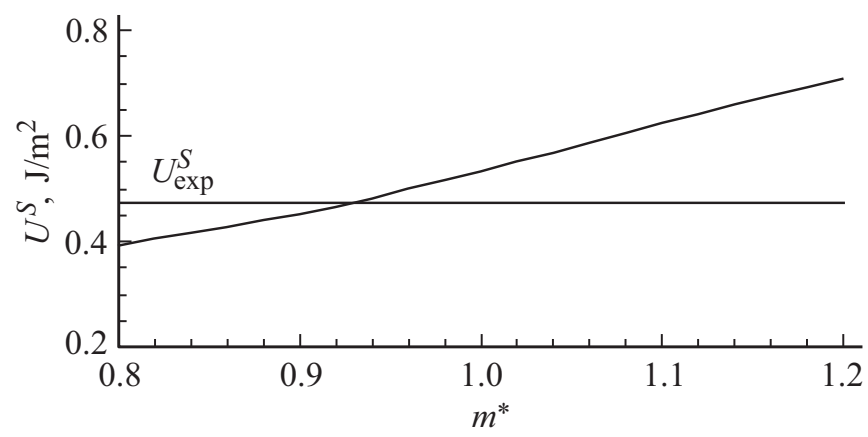

Рис. 3. Расчетная поверхностная энергия жидкой ртути. ли дополнены данными работы [16], в которой рабочий диапазон был расширен до $4 \mathrm{eV}$.

При описании модельного спектра оптической проводимости в [12] каждый вклад в процессы поглощения фотонов был представлен в графическом представлении с помощью значений положения пика поглощения фотонов (энергии фотона $\Delta_{j}=\hbar \omega_{j}$ ), высоты пика (совпадающей с частотой плазменных колебаний $\left.\omega_{p j}\right)$ и его полуширины (т.е. частоты релаксации $\omega_{0 j}$ ). Здесь $j-$ номер вклада. Вклад в оптическую проводимость электропроводности модели Друде учитывается с индексом $j=0$. Все эти характеристики пика поглощения фотонов рассматриваются в зависимости от эффективной массы электронов $m_{\mathrm{ef}}$.

Из правила сумм можно сделать вывод, что величина вклада частоты плазменных колебаний $\omega_{p j}$ в оптическую проводимость во многом зависит от заселенности электронами уровня $z_{j}$, с которого совершается инициированный фотонами переход. На рис. 2 показана зависимость от эффективной массы электронов вкладов величин $\omega_{p j}$ в оптическую проводимость с $j$ от 0 до 2. Из него видно, что полоса проводимости расщеплена и в зоне проводимости электронов нет, поскольку $\omega_{p 0} \approx 0$. При описании электропроводности этого металла при комнатной температуре нет необходимости учитывать проводимость модели Друде. Стационарную проводимость ртути обеспечивают электроны, находящиеся в полосе с $j=1$.

В таблице приведены электронные параметры вкладов в модельный спектр оптической проводимости, обеспечивающие наилучшее совпадение его с данными эксперимента. Эти данные представлены для значения эффективной массы электронов, полученной из условия близости экспериментальных [17] и расчетных значений поверхностной энергии. Значение эффективной массы электронов для этого условия (рис. 3) близко к 0.94 . При расчете поверхностной энергии, согласно [18], суммируются два вклада от поверхностных локализованных состояний (учитываются электронные характеристики для полос с $j=2$ и 3 ).

Работа выполнена по теме гос. задания № AАAА-А16$116122810219-4$. 


\section{Список литературы}

[1] Mott N.F. // Phil. Mag. 1966. V. 13. N 125. P. 989.

[2] Norris C., Rodway D.C., Williams C.P., Enderby J.E. // J. Phys. F: Met. Phys. 1973. V. 3. N 10. P. L182.

[3] Cotti P., Guntherodt H.-J., Munz P. at al. // Solid Stat. Commun. 1973. V. 12. N 7. P. 635.

[4] Tsuji K., Fukushima J., Oshima R., Endo H. // Phys. Lett. A. 1969. V 30. N 3. P. 173

[5] Barton L.D., Huang W.F. // Phys. Lett. A. 1974. V. 49. N 4. P. 323.

[6] Gustafson D.R., Mackintosh A.R., Zaffarano D.J. // Phys. Rev. 1963. V. 130. N 4. P. 1455.

[7] Hensel F., Franck E.U. // Rev. Mod. Phys. 1968. V. 40. N 4. P. 697.

[8] Even U., Jortner J. // Phys. Rev. B. 1973. V. 8. N 6. P. 2536.

[9] Kresse G., Hafner J. // Phys. Rev. B. 1997. V. 55. N 12. P. 7539.

[10] Schulz L. // Adv. Phys. 1957. V. 6. N 21. P. 102.

[11] Hodson J.N. // Advances in Physics. 1967. V. 16. N 64. P. 675.

[12] Киселев А.И., Кононенко В.И., Акашев Л.А. // ЖТФ. 2007. T. 77. B. 10. C. 75.

[13] Киселев А.И., Кононенко В.И. // ТВТ. 2003. Т. 41. № 6. C. 870.

[14] Hodgson J.N. // Phil. Mag. 1959. V. 4. N 38. P. 183.

[15] Faber T.E. // JOSA. 1968. V. 58. N 1. P. 102.

[16] Inagaki T., Arakawa E.T., Williams M.W. // Phys. Rev. B. 1981. V. 23. N 10. P. 5246.

[17] Попель С.И. Поверхностные явления в расплавах. М.: Металлургия, 1994. 440 с.

[18] Киселев А.И., Акашев Л.А., Кононенко В.И. // ТВТ. 2004. Т. 42. № 5. С. 709 . 